\title{
Topology of the regular part for infinitely renormalizable quadratic polynomials
}

\author{
by \\ Carlos Cabrera (Cuernavaca) and Tomoki Kawahira (Nagoya)
}

\begin{abstract}
We describe the well studied process of renormalization of quadratic polynomials from the point of view of their natural extensions. In particular, we describe the topology of the inverse limit of infinitely renormalizable quadratic polynomials and prove that when they satisfy a priori bounds, the topology is rigid modulo combinatorial equivalence.
\end{abstract}

1. Introduction and basic theory. There are some dynamical systems that can be interpreted by geometric objects. Sullivan constructed a lamination by Riemann surfaces associated to expanding maps on the circle, by using its inverse limit [20]. These laminations play a crucial role in his proof of the universality of infinitely renormalizable unimodal maps. Later on in [16], Lyubich and Minsky generalized this construction to every rational map on the sphere. They proved a rigidity theorem for critically non-recurrent rational maps without parabolic cycles, by means of hyperbolic 3-laminations associated to these maps.

For a given rational map, Lyubich and Minsky's construction of the hyperbolic 3-lamination is essentially based on the construction of a Riemann surface lamination associated to the map. In their setting, they also used inverse limits. However, the construction of the Riemann surface lamination is more involved than Sullivan's, since the presence of critical orbits forces some "irregular points" to be excluded from the inverse limit. The rest is called the regular part (or regular leaf space), which has nice analytic properties. Indeed, we may regard the regular part as the desired Riemann surface lamination when the critical orbits behave nicely.

Part of the program presented by Lyubich and Minsky was to investigate the properties of the regular part of quadratic maps $f_{c}(z)=z^{2}+c([16, \S 10])$.

2010 Mathematics Subject Classification: 37F10, 37F25, 37F45.

Key words and phrases: quadratic polynomial, Riemann surface lamination. 
Here are two possible problems:

- Describe the topological structure of the regular part for a quadratic map.

- Classify the quadratic maps by the topologies of their regular parts.

The authors dealt with these problems when $f_{c}$ has a (super)attracting or parabolic cycle and perhaps we have reasonable solutions [2, 3, 9, 10]. However, needless to say, the most important class to consider is the family of infinitely renormalizable quadratic maps.

Main results. Let us roughly summarize the main results of this paper. (The precise definitions and statements will be given later.) Let $f_{c}$ be an infinitely renormalizable quadratic map. Such a map uniquely determines an invariant called the combinatorics, which is represented by a sequence of parameters $\left\{s_{0}, s_{1}, \ldots\right\}$ with superattracting $f_{s_{i}}$. We say $f_{c}$ has a priori bounds when each level of renormalization is separated by an annulus of definite modulus.

Under the assumption of a priori bounds, the regular part of $f_{c}$ is a lamination under the topology induced from its inverse limit. Our results reveal the relations between the topology of the regular part $\mathcal{R}_{c}$ and the combinatorics $\left\{s_{0}, s_{1}, \ldots\right\}$ of $f_{c}$ with a priori bounds. The first theorem is:

STRUCTURE TheOREM. The regular part $\mathcal{R}_{c}$ of $f_{c}$ with a priori bounds is decomposed into blocks that are homeomorphic to the regular part of $f_{s_{i}}$ for $s_{i}$ in the combinatorics of $f_{c}$. In particular, the configuration of the blocks perfectly reflects the nest of the renormalizations.

Next we consider the classification of such an $f_{c}$ by the topology of regular parts. We will prove:

MaIn TheOREM. If two non-real infinitely renormalizable maps $f_{c}$ and $f_{c^{\prime}}$ with a priori bounds admit an orientation preserving homeomorphism between their regular parts, then they have the same combinatorics. Moreover, if the Mandelbrot set is locally connected at the parameter $c$, we have $c=c^{\prime}$.

Thus the topology of the regular part of the infinitely renormalizable map with a priori bounds has rigidity up to combinatorial equivalence. In particular, it may even determine the original dynamics if the Mandelbrot set is locally connected (cf. Mostow's rigidity for finite volume complete hyperbolic manifolds and corresponding Kleinian groups). The assumption of "non-real" is a technical condition for the proof, which we believe is unnecessary. On the other hand, the "orientation preserving" condition excludes trivial homeomorphisms that come from complex conjugation. 
Outline of the paper. In the rest of this section we give a brief introduction to the theory of dynamics of quadratic maps and their renormalizations. In Section 2, we review the definition of the inverse limits and the regular parts generated by quadratic maps. Section 3 is devoted to the statement and proof of the Structure Theorem (Theorem 4). Finally, in Section 4, we prove the Main Theorem (Theorem 7) stated as above.

1.1. Preliminaries. We start with notation on the dynamics of quadratic maps. Readers may refer to [4] and [13] for basic definitions.

Julia/Fatou sets, and external rays. For a quadratic map $f_{c}(z)=z^{2}+c$ on the Riemann sphere $\overline{\mathbb{C}}$, we denote the Julia set by $J\left(f_{c}\right)$, and the Fatou set by $F\left(f_{c}\right)$. The filled Julia set is denoted by $K\left(f_{c}\right)$. The closure of the forward orbit of 0 , denoted by $P\left(f_{c}\right)$, is called the postcritical set.

Throughout this paper we assume that $K\left(f_{c}\right)$ and $J\left(f_{c}\right)$ are both connected, i.e., the postcritical set $P\left(f_{c}\right)$ is bounded. Let $A_{c}:=\overline{\mathbb{C}} \backslash K\left(f_{c}\right)$ denote the basin of infinity of $f_{c}$. The Böttcher coordinate $\psi_{c}: A_{c} \rightarrow \overline{\mathbb{C}} \backslash \overline{\mathbb{D}}$ is the unique Riemann map with $\psi_{c}\left(f_{c}(z)\right)=\psi_{c}(z)^{2}$.

For $r>1$, the set $E_{c}(r):=\psi_{c}^{-1}(\{w \in \mathbb{C}:|w|=r\})$ is called the equipotential curve of level $r$. For $\theta \in \mathbb{R} / \mathbb{Z}$, the set $R_{c}(\theta):=\psi_{c}^{-1}(\{w \in \mathbb{C} \backslash \overline{\mathbb{D}}$ : $\arg w=\theta\}$ ) is called the external ray of angle $\theta$.

Ray portrait. (See Milnor's [19, §6].) Let $O=\left\{p_{1}, \ldots, p_{m}\right\}$ be a repelling cycle of $f_{c}$. Let $\Theta\left(p_{i}\right)$ denote the set of angles of external rays landing at $p_{i}$. The collection $\operatorname{rp}(O)=\left\{\Theta\left(p_{1}\right), \ldots, \Theta\left(p_{m}\right)\right\}$ is called the ray portrait of $O$. A ray portrait is called non-trivial if there are at least two rays landing at every point in $O$.

Superattracting quadratic maps. When $f_{s}(z)=z^{2}+s$ has a cycle containing the critical point $z=0$, we say $s$ is a superattracting parameter. Let $\left\{\alpha_{s}(1), \ldots, \alpha_{s}(m)=0\right\}$ denote the superattracting cycle with $f_{s}\left(\alpha_{s}(i)\right)=$ $\alpha_{s}(i+1)$, where we take indices modulo $m$. Let $D_{s}$ be the Fatou component containing 0 . It is known that the dynamics of $f_{s}^{m}: \bar{D}_{s} \rightarrow \bar{D}_{s}$ is conjugate to $f_{0}: \overline{\mathbb{D}} \rightarrow \overline{\mathbb{D}}$. Let $\Psi_{s}: \bar{D}_{s} \rightarrow \overline{\mathbb{D}}$ be this conjugacy. The internal equipotential $I_{s}(r)$ of level $r<1$ is defined by $\Psi_{s}^{-1}(\{|w|=r\})$. We also denote $\Psi_{s}^{-1}(\{|w|<r\})$ by $D_{s}(r)$.

Let $O_{s}$ be the repelling cycle in $\bigcup_{1 \leq i \leq m} f_{s}^{i}\left(\partial D_{s}\right)$ that is the orbit of the pull-back of $1 \in \partial \mathbb{D}$ by $\Psi_{s}$. It is known that if $m \geq 2$, the ray portrait $\operatorname{rp}\left(O_{s}\right)$ is non-trivial. Indeed, a result due to Milnor (see [19]) states that the map $f_{s}$ is uniquely determined by $\operatorname{rp}\left(O_{s}\right)$. Following Milnor's terminology, we call $\operatorname{rp}\left(O_{s}\right)$ the characteristic ray portrait of $f_{s}$.

Quadratic-like maps. (See [13, 17, 18].) Let $g: U \rightarrow V$ be a quadraticlike map. Throughout this paper we will only consider quadratic-like maps $g: U \rightarrow V$ with connected filled Julia set $K(g)$. Let $J(g)$ and $P(g)$ denote 
the Julia set and the postcritical set of $g$. The $\beta$-fixed point of $g$ is denoted by $\beta(g)$.

By Douady-Hubbard's Straightening Theorem [4], there exists a unique $c=c(g) \in \mathbb{C}$ and a quasiconformal map $h: V \rightarrow V^{\prime}$ such that $h$ conjugates $g: U \rightarrow V$ to $f_{c}: f_{c}{ }^{-1}\left(V^{\prime}\right) \rightarrow V^{\prime}$ where $\bar{\partial} h=0$ a.e. on $K(g)$. The quadratic map $f_{c}$ is called the straightening of $g$ and $h$ is called a straightening map. Although such an $h$ is not uniquely determined, we always assume that any quadratic-like map $g$ comes together with one fixed straightening map $h=h_{g}$.

One can always find $r_{g}>1$ such that if $r$ satisfies $1<r \leq r_{g}$ and $\theta \in \mathbb{R} / \mathbb{Z}$, the pulled-back equipotential

$$
E_{g}(r):=h^{-1}\left(E_{c}(r)\right)
$$

and the external ray

$$
R_{g}(\theta):=h^{-1}\left(\left\{\rho e^{2 \pi i \theta}: 1<\rho \leq r_{g}\right\}\right)
$$

are well-defined.

Renormalization of quadratic maps. A quadratic-like map $g: U \rightarrow V$ is said to be renormalizable, if there exist a number $m>1$, called the order of renormalization, and two open sets $U_{1} \subset U$ and $V_{1} \subset V$ containing the critical point of $g$, such that $g_{1}=g^{m}: U_{1} \rightarrow V_{1}$ is again a quadratic-like map with connected Julia set $K\left(g_{1}\right)$. We say $g_{1}: U_{1} \rightarrow V_{1}$ is a renormalization of $g: U \rightarrow V$. We call $K_{1}:=K\left(g_{1}\right), g\left(K_{1}\right), \ldots, g^{m-1}\left(K_{1}\right)$ the little Julia sets. We also assume that $m$ is the minimal order with this property and that the renormalization is non-crossing. (See [17] or [18.)

Infinitely renormalizable maps. (See [15.) In this paper we only deal with quadratic-like maps which are restrictions of some iterated quadratic map. Given a quadratic map $f_{c}$ and $r>1$, let $U_{c}(r)$ denote the topological disk in $\mathbb{C}$ enclosed by $E_{c}(r)$; then the restriction $f_{c}: U_{c}(\sqrt{r}) \rightarrow U_{c}(r)$ is a quadratic-like map. Set $g_{0}=f_{c}, U_{0}:=U_{c}(\sqrt{r})$ and $V_{0}:=U_{c}(r)$. We say $f_{c}$ is infinitely renormalizable if there exist an infinite sequence of numbers $1=p_{0}<p_{1}<p_{2}<\cdots$ and two sequences of open sets $\left\{U_{n}\right\}$ and $\left\{V_{n}\right\}$ such that each $g_{n}=f_{c}^{p_{n}}: U_{n} \rightarrow V_{n}$ is a quadratic-like map, with the property that $g_{n+1}$ is a renormalization of $g_{n}$ of order $m_{n}:=p_{n+1} / p_{n}>1$. The index $n$ of $g_{n}$ is called the level of renormalization.

Combinatorics of renormalizable maps. (See Lyubich's [14] and [15].) From now on, $f_{c}$ will denote an infinitely renormalizable quadratic map with renormalizations $\left\{g_{n}: U_{n} \rightarrow V_{n}\right\}$ as above. In order to describe the combinatorics of $f_{c}$, first we observe that the orbit under $g_{n}$ of the $\beta$-fixed point of $g_{n+1}$ is a repelling cycle $O_{n}$ of $g_{n}$. 
By our non-crossing assumption, the ray portrait $\operatorname{rp}\left(h_{n}\left(O_{n}\right)\right)$ is nontrivial and it determines a unique superattracting quadratic map $f_{s_{n}}(z)=$ $z^{2}+s_{n}$ with characteristic ray portrait $\operatorname{rp}\left(h_{n}\left(O_{n}\right)\right)$. We call the infinite sequence of superattracting parameters $\left\{s_{0}, s_{1}, s_{2}, \ldots\right\}$ the combinatorics of $f_{c}$; this is a well-defined invariant if we take $m_{n}$ minimal for each $n$.

A priori bounds. An infinitely renormalizable $f_{c}$ is said to have a priori bounds if there exists $\epsilon>0$ such that $\bmod \left(V_{n} \backslash U_{n}\right)>\epsilon$ for all $n \geq 0$. As we will see later, this condition ensures nice properties of $P\left(f_{c}\right)$.

2. Inverse limits and regular parts. In this section we consider the inverse limit of a quadratic polynomial and define its regular part. In some sense, the regular part is the analytically well-behaved subspace of the inverse limit of a quadratic polynomial. Readers may refer to [16] and [8] for more details on the objects defined here.

\subsection{Inverse limits and solenoidal cones}

Inverse limits. Consider $\left\{f_{-n}: X_{-n} \rightarrow X_{-n+1}\right\}_{n=1}^{\infty}$, a sequence of $d$-to-1 branched covering maps on the manifolds $X_{-n}$ with the same dimension. The inverse limit of this sequence is defined as

$\lim _{\longleftarrow}\left(f_{-n}, X_{-n}\right):=\left\{\hat{x}=\left(x_{0}, x_{-1}, x_{-2}, \ldots\right) \in \prod_{n \geq 0} X_{-n}: f_{-n}\left(x_{-n}\right)=x_{-n+1}\right\}$.

The space $\lim _{-n}\left(f_{-n}, X_{-n}\right)$ has a natural topology which is induced from the product topology in $\prod_{n \geq 0} X_{-n}$. The projection $\pi: \underset{\longleftarrow}{\lim }\left(f_{-n}, X_{-n}\right) \rightarrow X_{0}$ is defined by $\pi(\hat{x}):=x_{0}$.

EXAMPLE 1 (Natural extensions of quadratic maps). When all the pairs $\left(f_{-n}, X_{-n}\right)$ coincide with $\left(f_{c}, \overline{\mathbb{C}}\right)$, following Lyubich and Minsky [16] we will denote $\lim \left(f_{c}, \overline{\mathbb{C}}\right)$ by $\mathcal{N}_{c}$. The set $\mathcal{N}_{c}$ is called the natural extension of $f_{c}$. In this case, we denote the projection to the first coordinate by $\pi_{c}: \mathcal{N}_{c} \rightarrow \overline{\mathbb{C}}$. There is a natural homeomorphism $\hat{f}_{c}: \mathcal{N}_{c} \rightarrow \mathcal{N}_{c}$ given by $\hat{f}_{c}\left(z_{0}, z_{-1}, \ldots\right):=$ $\left(f_{c}\left(z_{0}\right), z_{0}, z_{-1}, \ldots\right)$.

Let $X$ be a forward invariant set. The invariant lift $\hat{X}$ of $X$ is the set of points $\hat{z} \in \mathcal{N}_{c}$ such that all coordinates of $\hat{z}$ belong to $X$. In particular, $\hat{\infty}=(\infty, \infty, \ldots)$.

Inverse limits appear naturally in dynamics, for instance, in the dynamics of Hénon maps. See [5] for more details.

EXAMPLE 2 (The dyadic solenoid). A well-known example of an inverse limit is the dyadic solenoid $\mathcal{S}^{1}:=\lim \left(f_{0}, \mathbb{S}^{1}\right)$, where $f_{0}(z)=z^{2}$ and $\mathbb{S}^{1}$ is the unit circle in $\mathbb{C}$. The dyadic solenoid is a connected set but is not path-connected. Since $\mathbb{S}^{1}$ is a topological group, $\mathcal{S}^{1}$ is a topological group 
acting on itself by translations: For any element $\tau=\left(\tau_{0}, \tau_{-1}, \ldots\right)$ in $\mathcal{S}^{1}$, the left translation $\tau: \mathcal{S}^{1} \rightarrow \mathcal{S}^{1}$ is given by $\tau\left(z_{0}, z_{-1}, \ldots\right)=\left(\tau_{0} z_{0}, \tau_{-1} z_{-1}, \ldots\right)$.

Solenoidal cones. A solenoidal cone is a space homeomorphic to $\lim \left(f_{0}, \overline{\mathbb{C}} \backslash \overline{\mathbb{D}}\right)$. Let $f_{c}$ be a map with connected $K\left(f_{c}\right)$; then the set $\hat{A}_{c}:=$ $\longleftarrow\left(f_{c}, A_{c}\right)$ in $\mathcal{N}_{c}$ is a solenoidal cone. To see this, consider the lift of the inverse $\psi_{c}^{-1}$ of the Böttcher coordinate given by $\hat{\psi}_{c}^{-1}:\left(z_{0}, z_{-1}, \ldots\right) \mapsto$ $\left(\psi_{c}^{-1}\left(z_{0}\right), \psi_{c}^{-1}\left(z_{-1}\right), \ldots\right)$. The set $\hat{A}_{c} \backslash\{\hat{\infty}\}$ is foliated by sets of the form $\mathcal{S}_{c}(r):=\pi_{c}^{-1}\left(E_{c}(r)\right)$ with $r>1$. For every $r, \mathcal{S}_{c}(r)$ is homeomorphic to the dyadic solenoid. In fact, there is a canonical homeomorphism $\phi_{r}=\phi_{c, r}$ : $\mathcal{S}_{c}(r) \rightarrow \mathcal{S}^{1}$ defined by $\phi_{r}:\left(z_{0}, z_{1}, \ldots\right) \mapsto\left(\psi_{c}\left(z_{0}\right) / r, \psi_{c}\left(z_{1}\right) / r^{1 / 2}, \ldots\right)$. We call such $\mathcal{S}_{c}(r)$ a solenoidal equipotential.

Let us give a few more examples of solenoidal cones. For $r>1$, set $\mathbb{D}_{r}:=\{|z|<r\}$. We denote the inverse limit associated with the backward dynamics

$$
\cdots \stackrel{f_{0}}{\longrightarrow} \overline{\mathbb{C}} \backslash f_{0}^{-2}\left(\overline{\mathbb{D}}_{r}\right) \stackrel{f_{0}}{\longrightarrow} \overline{\mathbb{C}} \backslash f_{0}^{-1}\left(\overline{\mathbb{D}}_{r}\right) \stackrel{f_{0}}{\longrightarrow} \overline{\mathbb{C}} \backslash \overline{\mathbb{D}}_{r}
$$

by $\hat{A}_{0}(r)$. This is a solenoidal cone compactly contained in $\hat{A}_{0} \subset \mathcal{N}_{0}$. Similarly, the set $\hat{A}_{c}(r):=\hat{\psi}_{c}^{-1}\left(\hat{A}_{0}(r)\right)$ is a solenoidal cone compactly contained in $\hat{A}_{c} \subset \mathcal{N}_{c}$. Note that the boundary of $\hat{A}_{c}(r)$ in $\mathcal{N}_{c}$ is $\mathcal{S}_{c}(r)$. The union $\hat{A}_{c}(r) \cup \mathcal{S}_{c}(r)$ is called the compact solenoidal cone at infinity of radius $r$ associated to $f_{c}$.

Let $f_{s}$ be a superattracting quadratic map with critical orbit of size $m$. For all $r<1$, the inverse limit given by the backward dynamics

$$
\cdots \stackrel{f_{c}^{m}}{\longrightarrow} D_{s}\left(r^{1 / 4}\right) \stackrel{f_{c}^{m}}{\longrightarrow} D_{s}\left(r^{1 / 2}\right) \stackrel{f_{c}^{m}}{\longrightarrow} D_{s}(r)
$$

is also a solenoidal cone. We denote it by $\lim _{(}\left(f_{s}^{m}, D_{s}(r)\right)$. We may con-

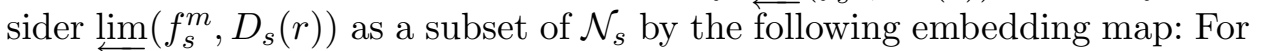
$\left.\left(x_{0}, x_{-1}, \ldots\right) \in \lim _{(}, f_{s}^{m}, D_{s}(r)\right)$, define $\iota:\left(x_{0}, x_{-1}, \ldots\right) \mapsto\left(y_{0}, y_{-1}, \ldots\right) \in \mathcal{N}_{s}$ so that $x_{-k}=y_{-m k}$ for all $k \geq 0$. Then $\hat{D}_{s}(r):=\iota\left(\lim \left(f_{s}^{m}, D_{s}(r)\right)\right)$ is a solenoidal cone in $\mathcal{N}_{s}$. Note that $\partial \hat{D}_{s}(r)$ is a proper subset of $\pi_{s}^{-1}\left(I_{s}(r)\right)$ unless $s=0$. Now $\hat{D}_{s}(r), \hat{f}_{s}\left(\hat{D}_{s}(r)\right), \ldots, \hat{f}_{s}^{m-1}\left(\hat{D}_{s}(r)\right)$ are disjoint solenoidal cones in $\mathcal{N}_{s}$.

Quadratic-like inverse limits. Let $g: U \rightarrow V$ be a proper holomorphic map, and let $\lim _{\longleftarrow}(g, V)$ denote the inverse limit for the sequence

$$
\cdots \rightarrow g^{-2}(V) \rightarrow g^{-1}(V) \rightarrow V .
$$

Here we allow $U=V$. In some cases $g$ will be taken as the restriction of a map defined on a larger set; in these cases we will consider all branches of the inverse of $g$ satisfying $g^{-n}(V) \subset U$.

We will use the following relation between inverse limits of quadraticlike maps and their straightenings: 
Proposition 1. Let $g: U \rightarrow V$ be a quadratic-like map with straightening $f_{c}(z)=z^{2}+c$. Then the inverse limit $\lim (g, V)$ is homeomorphic to $\mathcal{N}_{c}$ with a compact solenoidal cone at infinity removed.

Proof. Any topological conjugacy between two given maps induces a homeomorphism between the inverse limits. By the Straightening Theorem, $g$ is conjugated to a suitable restriction of $f_{c}$. By choosing the right conjugacy, $g$ is conjugated to $f_{c}$ restricted to the interior of some equipotential, say $f_{c}$ restricted to $U_{c}(\sqrt{r})$. Now, the inverse $\operatorname{limit} \lim \left(f_{c}, U_{c}(r)\right)$ is equal to $\mathcal{N}_{c}$ with the compact solenoidal cone of radius $r$ at infinity removed.

REMARK. By construction, the homeomorphism in Proposition 1 can be chosen to be a leafwise quasiconformal map.

\subsection{Regular parts and infinitely renormalizable maps}

Regular parts of quadratic natural extensions. Let $f_{c}$ be a quadratic map. A point $\hat{z}=\left(z_{0}, z_{-1}, \ldots\right)$, in the natural extension $\mathcal{N}_{c}=\lim _{(}\left(f_{c}, \overline{\mathbb{C}}\right)$, is called regular if there exists a neighborhood $U_{0}$ of $z_{0}$ such that the pull-back of $U_{0}$ along $\hat{z}$ is eventually univalent. The regular part (or regular leaf space) $\mathcal{R}_{f_{c}}=\mathcal{R}_{c}$ is the set of regular points in $\mathcal{N}_{c}$. Let $\mathcal{I}_{f_{c}}=\mathcal{I}_{c}$ denote the set of irregular points. Now we have $\mathcal{N}_{c}=\mathcal{R}_{c} \sqcup \mathcal{I}_{c}$.

The regular parts are analytically well-behaved subspaces of the natural extensions. More precisely, $\mathcal{R}_{c}$ is the largest subspace of $\mathcal{N}_{c}$ whose pathconnected components, called leaves, admit Riemann surface structures. It is a fact, due to Lyubich and Minsky [16], that all leaves of $\mathcal{R}_{c}$ are isomorphic to $\mathbb{C}$ or $\mathbb{D}$. Moreover, $\hat{f}_{c}$ sends leaves to leaves isomorphically. Leaves are wildly foliated in the natural extension: indeed, every leaf is dense in $\mathcal{N}_{c}$. See [16, §3] for more details.

EXAMPLE (Regular part of superattracting maps). Relevant examples of regular parts are given by superattracting quadratic maps. Let $f_{s}$ be a superattracting quadratic map with superattracting cycle $\left\{\alpha_{s}(1), \ldots, \alpha_{s}(m)=0\right\}$ as in the previous section. Under the homeomorphic action $\hat{f}_{s}: \mathcal{N}_{s} \rightarrow \mathcal{N}_{s}$, the points $\hat{\alpha}_{s}(i):=\left(\alpha_{s}(i), \alpha_{s}(i-1), \alpha_{s}(i-2), \ldots\right)$ form a cycle of period $m$. In this case, the set $\mathcal{I}_{s}$ of irregular points is $\left\{\hat{\infty}, \hat{\alpha}_{s}(1), \ldots, \hat{\alpha}_{s}(m)\right\}$. Thus the regular part $\mathcal{R}_{s}$ is $\mathcal{N}_{s}$ minus these $m+1$ irregular points. Note that for any $r>1, \hat{\infty} \in \hat{A}_{s}(r)$ and $\hat{\alpha}_{s}(i) \in \hat{f}_{s}^{i}\left(\hat{D}_{s}(1 / r)\right)$. Hence $\mathcal{I}_{s}$ is contained in a disjoint union of solenoidal cones. Under the topology induced from $\mathcal{N}_{s}, \mathcal{R}_{s}$ is a Riemann surface lamination with all leaves isomorphic to $\mathbb{C}$.

Regular part of infinitely renormalizable maps and a priori bounds. Let $\widehat{P\left(f_{c}\right)}$ be the invariant lift of the postcritical set, that is, the set of points $\hat{z}=\left(z_{0}, z_{-1}, \ldots\right) \in \mathcal{N}_{c}$ with $z_{-n} \in P\left(f_{c}\right)$ for all $n \geq 0$. 
Now suppose that $f_{c}$ has a priori bounds. If $K_{n}$ denotes the little Julia set of the $n$th renormalization, it follows that the postcritical set is given by

$$
P\left(f_{c}\right)=\bigcap_{n \geq 0} \bigcup_{j \geq 0} f_{c}^{j}\left(K_{n}\right)
$$

and homeomorphic to a Cantor set. Moreover, the map $f_{c}$ restricted to $P\left(f_{c}\right)$ acts as a minimal $\mathbb{Z}$-action. See McMullen's [17, Theorems 9.4]. Hence we have the following:

LEMMA 2. If $f_{c}$ is a quadratic polynomial with a priori bounds, then the set of irregular points in $\mathcal{N}_{c}-\{\hat{\infty}\}$ is $\widehat{P\left(f_{c}\right)}$. Moreover, the projection $\pi_{c}$ restricted to $\widehat{P\left(f_{c}\right)}$ is a homeomorphism over $P\left(f_{c}\right)$. Thus, the irregular part $\mathcal{I}_{c}$ is homeomorphic to a Cantor set together with the isolated point $\hat{\infty}$.

We will also need the following fact, due to Kaimanovich and Lyubich ([8, Lemma 3.18]):

TheOREM 3 (Kaimanovich-Lyubich). If $f_{c}$ has a priori bounds, then $\mathcal{R}_{c}$ is a locally compact Riemann surface lamination, whose leaves are conformally isomorphic to $\mathbb{C}$.

Theorem 3 is originally proved for $f_{c}$ with persistent recurrence, which is a weaker condition than a priori bounds.

3. Structure Theorem. In this section we describe in detail the topological structures of the natural extensions of infinitely renormalizable quadratic maps.

Blocks for superattracting maps. We first define the blocks associated with superattracting quadratic maps. Let $s$ be a superattracting parameter as in Section 1, with a superattracting cycle of period $m \geq 2$. For a fixed $r>1$, we set

$$
\mathcal{B}_{s}:=\mathcal{N}_{s} \backslash\left(\overline{\hat{A}_{s}(r)} \cup \bigsqcup_{i=0}^{m-1} \hat{f}_{s}^{i}\left(\overline{\hat{D}_{s}(1 / r)}\right)\right)
$$

and call it a block associated with $f_{s}$. That is, $\mathcal{B}_{s}$ is the natural extension of $f_{s}$ with compact solenoidal cones at each of the irregular points removed. Note that $\mathcal{B}_{s}$ is an open set and has $m+1$ boundary components which are all solenoidal equipotentials.

In addition, we also define

$$
\mathcal{Q}_{s}:=\mathcal{N}_{s} \backslash\left(\{\hat{\infty}\} \cup \bigsqcup_{i=0}^{m-1} \hat{f}_{s}^{i}\left(\overline{\hat{D}_{s}(1 / r)}\right)\right)=\mathcal{B}_{s} \sqcup\left(\overline{\hat{A}_{s}(r)} \backslash\{\hat{\infty}\}\right)
$$

for later use. 
Structure Theorem for infinitely renormalizable maps. Recall that the combinatorics of infinitely renormalizable maps are characterized by sequences of superattracting parameters. The following theorem shows that the natural extensions of infinitely renormalizable quadratic maps can be partially decomposed into blocks which are homeomorphic to the blocks of such superattracting parameters. We will establish:

TheOREM 4 (Structure Theorem). Let $f_{c}$ be infinitely renormalizable with a priori bounds, and $\left\{g_{n}=f_{c}^{p_{n}}: U_{n} \rightarrow V_{n}\right\}_{n>0}$ be the associated sequence of renormalizations with combinatorics $\left\{s_{0}, s_{1}, \ldots\right\}$. Set $m_{n}:=$ $p_{n+1} / p_{n}$. Then there exist disjoint open subsets $\mathcal{B}_{0}, \mathcal{B}_{1}, \mathcal{B}_{2}, \ldots$ of $\mathcal{R}_{c}$ such that:

(1) For $n=0$, the set $\mathcal{B}_{0}$ is homeomorphic to $\mathcal{Q}_{s_{0}}$. Moreover, the union $\mathcal{B}_{0} \cup\{\hat{\infty}\}$ forms a neighborhood of $\hat{\infty}$ with $m_{0}$ boundary components which are all homeomorphic to the dyadic solenoid.

(2) For each $n \geq 1$, the set $\mathcal{B}_{n}$ is homeomorphic to $\mathcal{B}_{s_{n}}$. Moreover, $\mathcal{B}_{n}$ has $m_{n}+1$ boundary components which are all homeomorphic to the dyadic solenoid.

(3) For any $n \geq 1$, the sets $\mathcal{B}_{n}, \hat{f}_{c}\left(\mathcal{B}_{n}\right), \ldots, \hat{f}_{c}^{p_{n}-1}\left(\mathcal{B}_{n}\right)$ have disjoint closures.

(4) For $0 \leq n<n^{\prime}$, the closures $\overline{\mathcal{B}_{n}}$ and $\overline{\mathcal{B}_{n^{\prime}}}$ intersect iff $n^{\prime}=n+1$. In this case, for all $0 \leq i<m_{n}$ the closures $\overline{\hat{f}_{c}^{i p_{n}}\left(\mathcal{B}_{n+1}\right)}$ and $\overline{\mathcal{B}_{n}}$ share just one of their solenoidal boundary components.

(5) The natural extension of $f_{c}$ and its regular part are given by

$$
\mathcal{N}_{c}=\{\hat{\infty}\} \sqcup \mathcal{R}_{c} \sqcup \widehat{P\left(f_{c}\right)} \quad \text { and } \quad \mathcal{R}_{c}=B_{0} \sqcup \bigcup_{n=1}^{\infty} \bigsqcup_{i=0}^{p_{n}-1} \overline{\hat{f}_{c}^{i}\left(\mathcal{B}_{n}\right)} .
$$

We call the open sets $\left\{\hat{f}_{c}^{i}\left(\mathcal{B}_{n}\right)\right\}_{n, i}$ blocks. Theorem 4 implies that $\mathcal{R}_{c}$ has a (locally finite) tree structure given by configuration of the blocks (Figure 3 below). Note that the block $\mathcal{B}_{n}$ which we will construct may not be an invariant set of $f_{c}^{p_{n}}$.

3.1. Proof of the Structure Theorem. We construct the blocks by an iterative procedure. Let us state it as a lemma so that we can apply it to each level of the renormalization.

Let $g: U \rightarrow V$ be an infinitely renormalizable quadratic-like map with combinatorics $\left\{s=s_{0}, s_{1}, s_{2}, \ldots\right\}$ and a renormalization $g_{1}=g^{m}: U_{1} \rightarrow V_{1}$. For $r>1$, let $U_{g}(r)$ denote the topological disk in $\mathbb{C}$ enclosed by $E_{g}(r)$. We may assume that $V=U_{g}(r)$ and $V_{1}=U_{g_{1}}\left(r_{1}\right)$ for some $r, r_{1}>1$, and $r_{1}$ is so close to 1 that

$$
U_{1} \Subset V_{1} \Subset g^{-m}(V) \Subset U \Subset V .
$$


Since $V_{1} \Subset g^{-m}(V)$, the map $\left.g^{i}\right|_{V_{1}}$ makes sense and $g^{i}\left(V_{1}\right) \subset V$ for all $1 \leq i \leq m$.

Set $\mathcal{X}:=\lim (g, V)$ and $\mathcal{X}_{1}:=\lim \left(g_{1}, V_{1}\right)$. Then $\mathcal{X}_{1}$ is naturally embedded in $\mathcal{X}$ as follows: For $\hat{x}=\left(x_{0}, x_{-1}, \ldots\right) \in \mathcal{X}_{1}$, set $\iota(\hat{x}):=\left(x_{0}^{*}, x_{-1}^{*}, \ldots\right) \in \mathcal{X}$ so that $x_{-k}=x_{-m k}^{*}$ for all $k \geq 0$. We denote the embedded image $\iota\left(\mathcal{X}_{1}\right)$ by $\mathcal{X}_{1}^{*}$. By Proposition 1, $\mathcal{X}_{1}\left(\right.$ resp. $\left.\mathcal{X}_{1}^{*}\right)$ is homeomorphic to $\mathcal{N}_{c_{1}}$ with a compact solenoidal cone at infinity removed, where $c_{1}=c\left(g_{1}\right)$ is the straightening of the infinitely renormalizable $g_{1}$ with combinatorics $\left\{s_{1}, s_{2}, \ldots\right\}$. Recall that $s=s_{0}$ is a superattracting parameter, and its characteristic ray portrait $\operatorname{rp}\left(O_{s}\right)$ is given by the cyclic orbit of $\beta\left(g_{1}\right)$ (the $\beta$-fixed point of $g_{1}$ ) under $g$. In principle, the set $\mathcal{X} \backslash \bigsqcup_{i=0}^{m-1} \overline{\hat{g}^{i}\left(\mathcal{X}_{1}^{*}\right)}$ is a prototype for the block homeomorphic to $\mathcal{B}_{s}$. However, the sets $\overline{\hat{g}}^{i}\left(\mathcal{X}_{1}^{*}\right)$ may not be disjoint, and this would contradict properties (2) and (3) of the theorem. This case occurs when the little Julia sets touch at the $\beta$-fixed point. To avoid this situation, we need to deform $\mathcal{X}_{1}^{*}$ to a smaller set $\mathcal{Y}_{1}^{*}$ such that $\mathcal{X}_{1}^{*}$ and $\mathcal{Y}_{1}^{*}$ are homotopically homeomorphic. More precisely, we claim:

Lemma 5. There exists a topological disk $W_{1} \subset V_{1}$ with the following properties:

(a) The set $\mathcal{Y}_{1}:=\lim \left(g_{1}, W_{1}\right) \subset \mathcal{X}_{1}$ is homeomorphic to $\mathcal{X}_{1}$.

(b) Set $\mathcal{Y}_{1}^{*}:=\iota\left(\mathcal{Y}_{1}\right) \subset \mathcal{X}$. Then the sets $\hat{g}^{i}\left(\mathcal{Y}_{1}^{*}\right)(0 \leq i<m)$ have disjoint closures.

(c) The set $\mathcal{X} \backslash \bigsqcup_{i=0}^{m-1} \overline{\hat{g}^{i}\left(\mathcal{Y}_{1}^{*}\right)}$ is homeomorphic to the block $\mathcal{B}_{s}$.

Proof of (a) and (b). We will modify the standard idea of thickening of puzzle pieces (see for instance [18, Lemmas 1.5 and 1.6]) into thinning. Set $\beta_{1}:=\beta\left(g_{1}\right)$ and $K_{1}:=K\left(g_{1}\right)$. In the pulled-back external rays landing at $\beta_{1}$ by the straightening map $h=h_{g}$, there are two of such rays $R_{1}$ and $R_{2}$ such that $R_{1} \cup R_{2}$ separates any other rays landing at $\beta_{1}$ and $K_{1} \backslash\left\{\beta_{1}\right\}$. Analogously, for the preimage $\beta_{1}^{*}:=g_{1}^{-1}\left(\left\{\beta_{1}\right\}\right) \backslash\left\{\beta_{1}\right\}$, there are two rays $R_{3}$ and $R_{4}$ landing at $\beta_{1}^{*}$ with the same property. The rays $\left\{R_{1}, R_{2}, R_{3}, R_{4}\right\}$ are called the supporting rays of $K_{1}$.

Next we take a small disk, centered at $\beta_{1}$, whose preimage under $g_{1}$ consists of two disks $\Delta$ and $\Delta^{*}$ around $\beta_{1}$ and $\beta_{1}^{*}$ respectively. The union of $\Delta, \Delta^{*}$, and the supporting rays divides $V$ into three topological disks. Let us take the one containing $K_{1} \backslash\left(\bar{\Delta} \cup \overline{\Delta^{*}}\right)$. By cutting the boundary by other external rays, we have a smaller domain $W^{\prime}$ shown in Figure 1 .

Let $W_{1}$ denote the topological disk that is the connected component of $W^{\prime} \cap V_{1}$ containing the critical point of $g_{1}$. Since $W_{1} \subset V_{1} \Subset g^{-m}(V)$, the sets $W_{1}, g\left(W_{1}\right), \ldots, g^{m-1}\left(W_{1}\right)$ are all defined. In particular, they have disjoint closures by construction. Now the inverse limit of the family $\left\{g_{1}: g_{1}^{-n-1}\left(W_{1}\right) \rightarrow g_{1}^{-n}\left(W_{1}\right)\right\}_{n \geq 0}$, which we denote by $\mathcal{Y}_{1}:=\varliminf_{\longleftarrow}\left(g_{1}, W_{1}\right)$, is a proper subset of $\mathcal{X}_{1}=\lim _{\longleftarrow}\left(g_{1}, V_{1}\right)$. 


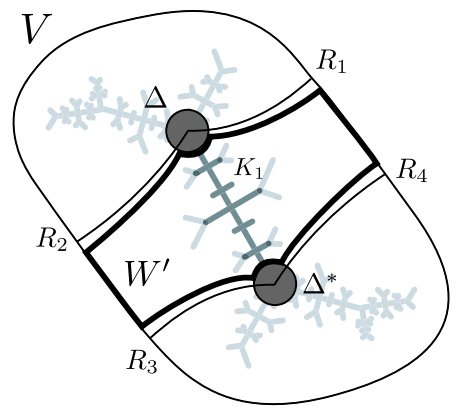

Fig. 1. The heavy curves show the boundary of $W^{\prime}$.

Let us check that $\mathcal{Y}_{1}$ is homeomorphic to $\mathcal{X}_{1}$. By definition $V_{1} \backslash W_{1}$ consists of disjoint topological disks and does not intersect the postcritical set $P\left(g_{1}\right)$ since we take sufficiently small $\Delta$ and $\Delta^{*}$. (See [17, Theorem 8.1] for example. This is the only part where we use the infinite renormalizability.) Thus $g_{1}: g_{1}^{-n-1}\left(V_{1}\right) \rightarrow g_{1}^{-n}\left(V_{1}\right)$ is isotopic to $g_{1}: g_{1}^{-n-1}\left(W_{1}\right) \rightarrow g_{1}^{-n}\left(W_{1}\right)$ for each $n \geq 0$, and this isotopy gives a homeomorphism between the inverse limits.

Let $\mathcal{Y}_{1}^{*}$ be the embedded image of $\mathcal{Y}_{1}$ under the map $\iota: \mathcal{X}_{1} \rightarrow \mathcal{X}$. For all $0 \leq i<m$, the sets $\hat{g}^{i}\left(\mathcal{Y}_{1}^{*}\right)$ are defined and have disjoint closures since their projections $g^{i}\left(W_{1}\right)$ are defined and have disjoint closures. Hence we have (a) and (b) of the lemma.

Proof of $(c)$. Set $\mathcal{B}:=\mathcal{X} \backslash \bigsqcup_{i=0}^{m-1} \hat{g}^{i}\left(\overline{\mathcal{Y}_{1}^{*}}\right)$. Now it is enough to show that $\mathcal{B}$ is homeomorphic to the block $\mathcal{B}_{s}$ associated with $f_{s}$, that is,

$$
\mathcal{B}_{s}=\mathcal{N}_{s} \backslash\left(\overline{\hat{A}_{s}(r)} \sqcup \bigsqcup_{i=0}^{m-1} \hat{f}_{s}^{i}\left(\overline{\hat{D}_{s}(1 / r)}\right)\right)=\pi_{s}^{-1}\left(U_{s}(r)\right) \backslash \bigsqcup_{i=0}^{m-1} \hat{f}_{s}^{i}\left(\overline{\hat{D}_{s}(1 / r)}\right) .
$$

Here we take the same $r$ as in the construction of $V=U_{g}(r)$. For later use we also set $V_{s}:=U_{s}(r)$.

We first work with the dynamics downstairs. Set $B:=V \backslash \bigsqcup_{i=0}^{m-1} g^{i}\left(\overline{W_{1}}\right)$ and mark $B$ with some arcs given as follows (see Figure 2, left): First join $g\left(\beta_{1}\right)$ and $\partial g\left(W_{1}\right)$ by an arc $\delta$ within $g(\Delta)$. Since $g: W_{1} \rightarrow g\left(W_{1}\right)$ is a branched covering, the pull-back $g^{-1}(\delta)$ has two components in $\Delta$ and $\Delta^{*}$. Now the markings are given by $g^{-1}(\delta), \delta, g(\delta), \ldots, g^{m-2}(\delta)$ and all of the forward images of the supporting rays $\bigcup_{j=1}^{4} R_{j}$. The markings decompose $B$ into finitely many pieces that are all topological disks. Note that the boundary of each piece intersects the equipotential $E_{g}(r)$ and at least two external rays.

Correspondingly, set $B_{s}:=V_{s} \backslash \bigsqcup_{i=1}^{m-1} f_{s}^{i}\left(\overline{D_{s}(1 / r)}\right)$, and complete the marking of $B_{s}$ by taking all the forward images of supporting rays of $D_{s}$ 
and small arcs from each point of the landing points of such rays to the equipotentials $f_{s}^{i}\left(I_{s}(1 / r)\right)$ (Figure 2 , right). The markings also decompose $B_{s}$ into some pieces as in the case of $B$.
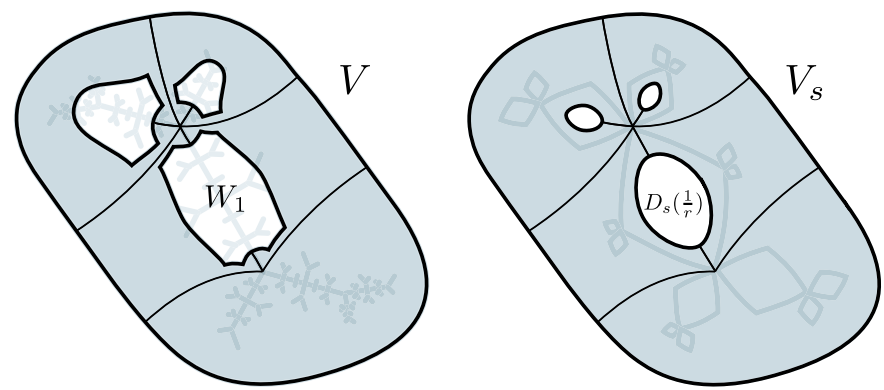

Fig. 2. The shaded regions show $B$ and $B_{s}$ with their markings drawn in.

Clearly, there is a homeomorphism $\phi$ from $B$ to $B_{s}$ respecting the configuration of the markings which in particular sends the supporting external rays to the supporting external rays without changing angles. Since by construction $B$ and $B_{s}$ do not intersect the postcritical sets, it is not difficult to check that the map $\phi$ lifts to a homeomorphism $\hat{\phi}: \pi^{-1}(B) \rightarrow \pi_{s}^{-1}\left(B_{s}\right)$ respecting the backward orbits of the markings, and then extends to a homeomorphism $\hat{\phi}: \mathcal{B} \rightarrow \mathcal{B}_{s}$.

Proof of Theorem 4 (Structure Theorem). We first set $\mathcal{X}_{0}=\lim _{(}\left(g_{0}, V_{0}\right) \subset$ $\mathcal{N}_{c}$ and apply Lemma 5 with $g=g_{0}$. Then we have a block $\mathcal{B}_{0}:=\mathcal{X}_{0} \backslash$ $\bigsqcup_{i=0}^{m_{0}-1} \hat{g}_{0}^{i}\left(\overline{\mathcal{Y}_{1}^{*}}\right)$ that is homeomorphic to $\mathcal{B}_{s_{0}}$. To get property $(1)$, we need to replace $\mathcal{B}_{0}$ by $\mathcal{R}_{c} \backslash \bigsqcup_{i=0}^{m-1} \overline{\hat{g}^{i}\left(\mathcal{Y}_{1}^{*}\right)}$ to cover all the basin at infinity of $f_{c}$. By Proposition 1 again, $\mathcal{B}_{0}$ is homeomorphic to $\mathcal{Q}_{s_{0}}$.

Now assume that for $n \geq 1$ we applied Lemma 5 with $g=g_{n-1}$, and we have a topological disk $W_{n} \subset V_{n}$ and $\mathcal{Y}_{n}=\lim _{\longleftarrow}\left(g_{n}, W_{n}\right) \subset \mathcal{X}_{n}=\lim _{\longleftarrow}\left(g_{n}, V_{n}\right)$. For the next induction step, we apply the lemma with a slight modification. We may assume that $g_{n}: U_{n} \rightarrow V_{n}$ and $g_{n+1}: U_{n+1} \rightarrow V_{n+1}$ satisfy the original condition

$$
U_{n+1} \Subset V_{n+1} \Subset g_{n}^{-m_{n}}\left(V_{n}\right) \Subset U_{n} \Subset V_{n}
$$

in the lemma, and also

$$
V_{n+1} \sqcup g_{n}\left(V_{n+1}\right) \sqcup \cdots \sqcup g_{n}^{m_{n}-1}\left(V_{n+1}\right) \Subset W_{n} .
$$

In the same way as in the lemma, we construct a topological disk $W_{n+1} \subset$

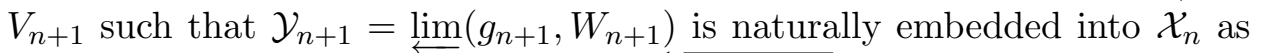
$\iota\left(\mathcal{Y}_{n+1}\right)=\mathcal{Y}_{n+1}^{*}$, and the set $\mathcal{X}_{n} \backslash \bigsqcup_{i=0}^{m_{n}-1} \overline{\hat{g}_{n}^{i}\left(\mathcal{Y}_{n+1}^{*}\right)}$ is homeomorphic to $\mathcal{B}_{s_{n}}$. In addition, the set $\mathcal{B}_{n}^{\prime}:=\mathcal{Y}_{n} \backslash \bigsqcup_{i=0}^{m_{n}-1} \overline{\hat{g}_{n}^{i}\left(\mathcal{Y}_{n+1}^{*}\right)}$ is also homeomorphic to $\mathcal{B}_{s_{n}}$, 
since $\mathcal{Y}_{n}$ is homeomorphic to $\mathcal{X}_{n}$ and we have

$$
\mathcal{Y}_{n+1}^{*} \sqcup \hat{g}_{n}\left(\mathcal{Y}_{n+1}^{*}\right) \sqcup \cdots \sqcup \hat{g}_{n}^{m_{n}-1}\left(\mathcal{Y}_{n+1}^{*}\right) \Subset \mathcal{Y}_{n} \subset \mathcal{X}_{n}
$$

by the second condition above.

Finally, we define the block $\mathcal{B}_{n}$ in the theorem by the image of the iterated natural embeddings

$$
\mathcal{B}_{n}^{\prime} \hookrightarrow \lim _{\longleftarrow}\left(g_{n-1}, W_{n-1}\right) \hookrightarrow \cdots \hookrightarrow \lim _{\longleftarrow}\left(g_{1}, W_{1}\right) \hookrightarrow \lim _{\longleftarrow}\left(g_{0}, V_{0}\right)
$$

so that the block becomes a subset of the original natural extension $\mathcal{N}_{c}$ (Figure 3). Now $\overline{\mathcal{B}_{n}}$ contains no irregular point by this construction. Hence $\overline{\mathcal{B}_{n}} \subset \mathcal{R}_{c}$. Properties (2), (3), and (4) of the theorem are clear, also by the construction.

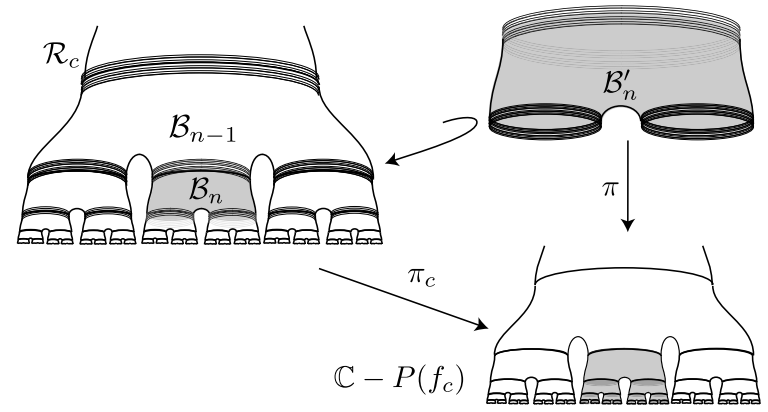

Fig. 3. A caricature of the tree structure of $\mathcal{R}_{c}$.

Only for (5) do we need the condition that $f_{c}$ has a priori bounds: By Lemma 2, the set $\widehat{P\left(f_{c}\right)} \cup\{\hat{\infty}\}$ consists of all the irregular points. Hence the regular part consists of all the backward orbits that do not remain in $P\left(f_{c}\right) \cup\{\infty\}$. Since such a backward orbit must be contained in either $\mathcal{B}_{0}$ or the closure of the block $\overline{\hat{f}_{c}^{i}\left(\mathcal{B}_{n}\right)}$ for some $n \geq 1$ and $0 \leq i<p_{n}$, we have $\mathcal{R}_{c} \subset B_{0} \sqcup \bigcup_{n=1}^{\infty} \bigsqcup_{i=0}^{p_{n}-1} \overline{\hat{f}_{c}^{i}\left(\mathcal{B}_{n}\right)}$. The opposite inclusion holds since $\overline{\mathcal{B}_{n}} \subset \mathcal{R}_{c}$ for all $n \geq 0$, thus the second equality in (5) follows. Now the first equality is straightforward.

To end this section we show a proposition that is important for the arguments in the next section.

Buildings at finite levels. For $f_{c}$ with a priori bounds, we define an open set

$$
\mathcal{Q}_{n}:=\mathcal{R}_{c} \backslash \bigcup_{k=n+1}^{\infty} \bigsqcup_{i=0}^{p_{k}-1} \overline{\hat{f}_{c}^{i}\left(\mathcal{B}_{k}\right)},
$$

which consists of blocks up to the $n$th level. Then any $\hat{z} \in \mathcal{R}_{c}$ is contained in $\mathcal{Q}_{n}$ with sufficiently large $n$. 
The proposition below states that $\mathcal{Q}_{n}$ can be embedded into the regular part of a superattracting quadratic map. For the combinatorics $\left\{s_{0}, s_{1}, \ldots\right\}$ of $f_{c}$, its finite subsequence $\left\{s_{0}, s_{1}, \ldots, s_{n}\right\}$ determines a superattracting parameter $\sigma_{n}$. More precisely, for a $\beta$-fixed point $\beta\left(g_{n+1}\right)$ of $g_{n+1}=f_{c}^{p_{n+1}}$, its forward orbit $O_{n+1}$ under $f_{c}$ forms a repelling periodic cycle. Then its ray portrait $\operatorname{rp}\left(O_{n+1}\right)$ uniquely determines a superattracting quadratic map $f_{\sigma_{n}}$. Now we have:

Proposition 6. For infinitely renormalizable $f_{c}$, let $\mathcal{Q}_{n}$ be the set defined as above. Then we have a homeomorphism $h_{n}$ between $\mathcal{Q}_{n}$ and $\mathcal{Q}_{\sigma_{n}}$.

Proof. The proof is almost straightforward by Lemma 5. In fact, we can apply the same argument by setting $g:=g_{0}$ and $g_{1}:=g_{n+1}$.

4. Rigidity. In this section we prove the Main Theorem of the paper, which is the following:

TheOREM 7 (Main Theorem). Let $f_{c}$ and $f_{c^{\prime}}$ be infinitely renormalizable maps with a priori bounds. Assume $\operatorname{Im}(c) \neq 0$. If $h: \mathcal{R}_{c} \rightarrow \mathcal{R}_{c^{\prime}}$ is an orientation preserving homeomorphism, then $c$ and $c^{\prime}$ belong to the same combinatorial class.

There is a natural homeomorphism between $\mathcal{N}_{c}$ and $\mathcal{N}_{\bar{c}}$, so we avoid this inconsistency by requiring $h$ to be orientation preserving.

From the point of view of the parameter plane, it is known that $c$ is combinatorially rigid if and only if the Mandelbrot set is locally connected (MLC) at $c$. In view of that, our main theorem has the following corollary.

Corollary 8. Assume that $h: \mathcal{R}_{c} \rightarrow \mathcal{R}_{c^{\prime}}$ and $c$ are as in the Main Theorem. If in addition the Mandelbrot set is locally connected at $c$, then $c=c^{\prime}$.

In [15, Lyubich proved MLC for $f_{c}$ with a priori bounds satisfying a secondary limb condition. In this direction, recent papers by Kahn [6] and Lyubich [7] give a priori bounds for infinitely renormalizable parameters with special combinatorics. In all these parameters, the Mandelbrot set is locally connected.

The strategy to prove the Main Theorem is to show that any homeomorphism given in the statement admits a homeomorphic extension to the natural extensions, satisfying the properties of the following general theorem:

THEOREM 9. Let $c$ and $c^{\prime}$ be any pair of parameters such that there exists $h: \mathcal{N}_{c} \rightarrow \mathcal{N}_{c^{\prime}}$, an orientation preserving homeomorphism between natural extensions, such that:

(1) $h(\hat{\infty})=\hat{\infty}$,

(2) $h$ sends repelling leaves into repelling leaves.

Then $f_{c}$ and $f_{c^{\prime}}$ belong to the same combinatorial class. 
A leaf $L$ in $\mathcal{R}_{c}$ is called repelling if it contains a repelling periodic point of $\hat{f}_{c}$. Clearly, every repelling leaf is invariant under some iterate of $\hat{f}_{c}$, but the converse is not true in general. For instance, if $f_{c}$ has a parabolic cycle, there are invariant leaves of $\mathcal{R}_{c}$ without periodic points.

We will see that, for quadratic polynomials with a priori bounds, repelling leaves have particular topological properties. Hence any homeomorphism as in the Main Theorem must send repelling leaves into repelling leaves.

This was the approach in [3] (see also [2]) to prove rigidity for hyperbolic and complex semihyperbolic maps.

4.1. Proof of Theorem 9. There are several models describing the combinatorics of quadratic polynomials; a comprehensive text can be found in [1. In this paper, we are going to adopt the description given by rational laminations. Any quadratic polynomial $f_{c}$ with $c$ in the Mandelbrot set determines an equivalence relation in $\mathbb{Q} / \mathbb{Z}$, called the rational lamination of $f_{c}$. This equivalence relation is defined as follows: Given $\theta$ and $\theta^{\prime}$ in $\mathbb{Q} / \mathbb{Z}$, we say that $\theta \sim \theta^{\prime}$ if the external rays $R_{\theta}$ and $R_{\theta^{\prime}}$ land at the same point in the Julia set $J\left(f_{c}\right)$.

Given any equivalence relation $R$ in $\mathbb{Q} / \mathbb{Z}$ we may ask whether $R$ is the lamination associated to a quadratic polynomial. In [11], Kiwi gave necessary and sufficient conditions for $R$ to be the rational lamination associated to a $\operatorname{map} f_{c}$.

Two parameters $c_{1}$ and $c_{2}$ in the Mandelbrot set are combinatorially equivalent if $f_{c_{1}}$ and $f_{c_{2}}$ determine the same rational lamination. Clearly, this is an equivalence relation in the Mandelbrot set. The induced partition also coincides with the combinatorial invariants given by ray portraits for superattracting maps $f_{s}$, or the sequence $\left\{s_{1}, s_{2}, \ldots\right\}$ for infinitely renormalizable maps $f_{c}$.

We will need the following property of rational laminations:

Lemma 10. Let $R$ and $R^{\prime}$ be two rational laminations, and assume that there is $\theta \in \mathbb{Q} / \mathbb{Z}$ such that each class in $R^{\prime}$ is obtained by rotating a class in $R$ by angle $\theta$. Then $\theta=0(\bmod 1)$.

In the dynamical plane, if $p$ is a periodic point in the Julia set $J\left(f_{c}\right)$ then $p$ is the landing point of external rays which are periodic under $f_{c}$ (see [19]). Now, if the periodic lift $\hat{p}$ belongs to the regular part, then there are periodic lifted external rays landing at $\hat{p}$ in $L(\hat{p})$. Each of these lifted external rays will intersect a leaf of a solenoidal equipotential.

Let us consider the dyadic solenoid $\mathcal{S}^{1}=\left\lfloor\lim \left(f_{0}, \mathbb{S}^{1}\right) \subset \mathcal{R}_{0}\right.$. One can check that every repelling periodic point in $\mathcal{R}_{0}$ belongs to $\mathcal{S}^{1}$. By restriction, let us call a leaf $S$ in $\mathcal{S}^{1}$ repelling if $S$ is contained in a repelling leaf in $\mathcal{R}_{0}$. If a leaf 
$S \subset \mathcal{S}^{1}$ is invariant under some iterate of $\hat{f}_{0}$, then $S$ must be repelling. In other words, there is a one-to-one correspondence between repelling leaves in $\mathcal{S}^{1}$ and periodic points in $\mathbb{S}^{1}$ of $f_{0}$. With this fact and the lift of Böttcher's coordinate we can carry information on repelling periodic points of $f_{c}$ to repelling leaves in $\mathcal{R}_{c}$.

More precisely, let $L$ be a repelling leaf in $\mathcal{R}_{c}$ and let $\mathcal{S}_{c}(r)$ be some solenoidal equipotential. Consider the intersection $L \cap \mathcal{S}_{c}(r)$, which may consist of several leaves in $\mathcal{S}_{c}(r)$. Let $\phi$ be the canonical homeomorphism of $\mathcal{S}_{c}(r)$ with $\mathcal{S}^{1}$; then every leaf in $\phi\left(L \cap \mathcal{S}_{c}(r)\right)$ is repelling in $\mathcal{S}^{1}$. Hence, every leaf in $\phi\left(L \cap \mathcal{S}_{c}(r)\right)$ contains a periodic point in $\mathcal{S}^{1}$ by $\hat{f}_{0}$. In fact, the pull-back to $L$ of each of these periodic points is precisely the intersection of a periodic solenoidal external ray landing at the periodic point of $L$. As a consequence we have:

Lemma 11. Let $S_{1}$ and $S_{2}$ be two leaves in $\mathcal{S}_{c}(r)$. Then $S_{1}$ and $S_{2}$ belong to the same repelling leaf $L$ in $\mathcal{R}_{c}$ if and only if they intersect at periodic lifted external rays landing at the same point in $\pi^{-1}\left(J\left(f_{c}\right)\right) \cap \mathcal{R}_{c}$.

The proof of Theorem 9 is decomposed into three statements: Lemma 12 whose proof can be found in [3], Proposition 13 which is due to Kwapisz [12], and Lemma 14 . The first starts by noting that the foliation of the solenoidal cone by solenoidal equipotentials defines a local base of neighborhoods at $\hat{\infty}$ in $\mathcal{N}_{c}$. Hence, given a homeomorphism $h$ as in Theorem 9 , we can find a solenoidal equipotential $\mathcal{S}_{c}(r)$ whose image lies between two solenoidal equipotentials. Recall that a solenoidal equipotential $\mathcal{S}_{c}(r)$ has associated a canonical homeomorphism $\phi_{r}: \mathcal{S}_{c}(r) \rightarrow \mathcal{S}^{1}$, moreover, $\phi_{r^{2}} \circ \hat{f}_{c} \circ \phi_{r}^{-1}=\hat{f}_{0}$. So the map $h \circ \phi_{r}^{-1}$ maps $\mathcal{S}^{1}$ into a cylinder over $\mathcal{S}^{1}$ bounded by the two solenoidal equipotentials. Hence, we are in the following situation:

Lemma 12. Let e $: \mathcal{S}^{1} \rightarrow \mathcal{S}^{1} \times(0,1)$ be a topological embedding. Then there is an embedding $e^{\prime}: \mathcal{S}^{1} \rightarrow \mathcal{S}^{1} \times(0,1)$ isotopic to e such that $e^{\prime}\left(\mathcal{S}^{1}\right)=$ $\mathcal{S}^{1} \times\{1 / 2\}$.

The isotopy in Lemma 12 pulls back to an isotopy $\psi$ defined on $\mathcal{S}_{c}(r)$. This isotopy induces a homeomorphism $h^{\prime}$ from $\mathcal{S}_{c}(r)$ to a solenoidal equipotential in $\mathcal{N}_{c^{\prime}}$. We can extend $\psi$ to an isotopy defined globally in $\mathcal{N}_{c}$, so that this isotopy is the identity outside a neighborhood of $\mathcal{S}_{c}(r)$. With this construction, $h^{\prime}$ extends to a homeomorphism defined on $\mathcal{N}_{c}$ and isotopic to $h$.

We now restrict our attention to the map $h^{\prime} \mid \mathcal{S}_{c}(r)$. We want to study the isotopic properties of $h^{\prime} \mid \mathcal{S}_{c}(r)$ but keeping in mind that every map isotopic to $h^{\prime} \mid \mathcal{S}_{c}(r)$ will induce a map isotopic to $h^{\prime}$ defined globally in $\mathcal{R}_{c}$. Using the canonical homeomorphism of solenoidal equipotentials to $\mathcal{S}^{1}$, we see that $h^{\prime}$ restricted to $\mathcal{S}_{c}(r)$ induces a self-homeomorphism of the dyadic solenoid $\mathcal{S}^{1}$. Now by the group structure of $\mathcal{S}^{1}$, as described by Kwapisz in [12, each 
homotopy class of homeomorphisms of $\mathcal{S}^{1}$ is uniquely represented by a map with a special form:

Proposition 13 (Kwapisz). Let $\Phi: \mathcal{S}^{1} \rightarrow \mathcal{S}^{1}$ be a homeomorphism of the dyadic solenoid. Then there exist a unique $n$ and an element $\tau \in \mathcal{S}^{1}$ such that $\Phi$ is isotopic to $\hat{z} \mapsto \tau \hat{f}_{0}^{n}(\hat{z})$.

The number $n$ is uniquely determined by the homotopy class of $h^{\prime}$. The map $\hat{f}_{c^{\prime}}^{-1}$ restricted to a solenoidal equipotential acts, under canonical homeomorphism, in $\mathcal{S}^{1}$ as $\hat{f}_{0}^{-1}$. Note that if $\tau \in \mathcal{S}^{1}$, then $\hat{f}_{0}^{-1}(\tau \hat{z})=$ $\hat{f}_{0}^{-1}(\tau) \hat{f}_{0}^{-1}(\hat{z})$. Thus, by post-composing $h^{\prime}$ with $\hat{f}_{c^{\prime}}^{-n}$, Proposition 13 implies that $\hat{f}_{c^{\prime}}^{-n} \circ h^{\prime}$ is isotopic to a homeomorphism $h^{\prime \prime}: \mathcal{N}_{c} \rightarrow \mathcal{N}_{c^{\prime}}$ that sends a solenoidal equipotential, say $\mathcal{S}_{c}(r)$, into a solenoidal equipotential. The restriction of $h^{\prime \prime}$ to $\mathcal{S}_{c}(r)$, under the canonical homeomorphism to $\mathcal{S}^{1}$ is just some translation by $\tau^{\prime}:=\hat{f}_{0}^{-n}(\tau)$ of the dyadic solenoid $\mathcal{S}^{1}$.

Isotopies defined in solenoids keep each leaf invariant. On the other hand, the map $\hat{f}_{c^{\prime}}$ sends repelling leaves into repelling leaves. So, if $h$ is a homeomorphism that sends repelling leaves into repelling leaves, the map $h^{\prime \prime}$, induced by Proposition 13, also sends repelling leaves to repelling leaves.

Lemma 14. Let $h^{\prime \prime}$ be the homeomorphism above. Then $\tau^{\prime}$ is isotopic to the identity.

Proof. Let us consider the restriction of $h^{\prime \prime}$ to the solenoidal equipotential $\mathcal{S}_{c}(r)$ such that $h^{\prime \prime}\left(\mathcal{S}_{c}(r)\right)$ is also a solenoidal equipotential. Under canonical homeomorphisms we can regard $H:=h^{\prime \prime} \mid \mathcal{S}_{c}(r)$ as a self-map of $\mathcal{S}^{1}$. We assume that $H$ has the form $\hat{z} \mapsto \tau^{\prime} \hat{z}$. By Lemma 11, $H$ sends repelling leaves into repelling leaves.

Let $S$ be a periodic leaf in $\mathcal{S}^{1}$ with periodic point $\hat{\theta}$, and let $\hat{\theta}^{\prime}$ be the periodic point in $H(S)$. By sliding $\mathcal{S}^{1}$ along $H(S)$ to send $H(\hat{\theta})$ to $\hat{\theta}^{\prime}$, this operation induces a new map $H^{\prime}$ in the isotopy class of $H$, which satisfies $H^{\prime}(\hat{\theta})=\tau^{\prime \prime} \hat{\theta}=\hat{\theta}$ for some $\tau^{\prime \prime} \in \mathcal{S}^{1}$. Since $\hat{\theta}$ and $\hat{\theta}^{\prime}$ are periodic in $\mathcal{S}^{1}$, also $\tau^{\prime \prime}$ must be periodic. Hence, the map $H^{\prime}$ leaves the set of periodic points in $\mathcal{S}^{1}$ invariant.

Now, periodic points in $\mathcal{S}^{1}$ are determined by the first coordinate. The translation $\tau^{\prime}$ induces a rotation in the set of periodic angles which extends to a rotation on the rational lamination. By Lemma 10 this implies that the rational laminations are the same, and that the translation $\tau^{\prime \prime}$ is the identity. By construction, $\tau^{\prime}$ is isotopic to $\tau^{\prime \prime}$.

Proof of Theorem 9. From the existence of the homeomorphism $h$, we obtained the homeomorphism $h^{\prime \prime}$. In turn, Lemma 14 implies that $h^{\prime \prime}$ is isotopic to another homeomorphism. Such homeomorphism when restricted to a solenoidal equipotential is the identity, under canonical identifications. Any rational ray $R_{\theta}$ landing in $J\left(f_{c}\right)$ lifts to a periodic external ray in $\mathcal{N}_{c}$. 
Under canonical identification, the intersection of the lift of periodic external rays is a periodic point in $\mathcal{S}^{1}$. Moreover, this periodic point in $\mathcal{S}^{1}$ is precisely the periodic lift of angle $\theta$ in $\mathcal{S}^{1}$. Finally, Lemma 11 implies that the rational laminations of $f_{c}$ and $f_{c^{\prime}}$ are the same. Thus $c$ and $c^{\prime}$ belong to the same combinatorial class.

4.2. Ends of the regular part. A path $\gamma:[0, \infty) \rightarrow \mathcal{R}_{c}$ is said to escape to infinity if it leaves every compact set $K \subset \mathcal{R}_{c}$. We define an end of $\mathcal{R}_{c}$ to be an equivalence class of paths escaping to infinity. Let $\gamma$ and $\sigma$ be two paths escaping to infinity. We say that $\gamma$ and $\sigma$ access the same end if for every compact set $K \subset \mathcal{R}_{c}$, the paths $\gamma$ and $\sigma$ eventually belong to the same connected component of $\mathcal{R}_{c} \backslash K$. Consider the set $\operatorname{End}\left(\mathcal{R}_{c}\right)$ which is the union of $\mathcal{R}_{c}$ with the abstract set of ends.

Let $f_{c}$ be an infinitely renormalizable quadratic polynomial with a priori bounds. By Theorem 3, the regular part $\mathcal{R}_{c}$ is locally compact. Thus $\operatorname{End}\left(\mathcal{R}_{c}\right)$ is a compact set, which we will call the end compactification of $\mathcal{R}_{c}$.

Proposition 15. Let $f_{c}$ be an infinitely renormalizable quadratic polynomial with a priori bounds. Then $\operatorname{End}\left(\mathcal{R}_{c}\right)$ is homeomorphic to $\mathcal{N}_{c}$.

Proof. First, we will show that there exists a bijection $\Phi$ between the set of irregular points and the set of ends. Let $\hat{i}$ be an irregular point in $\mathcal{N}_{c}$, let $i_{0}=\pi(\hat{i})$ and take any $z_{0} \in \mathbb{C} \backslash P\left(f_{c}\right)$. Since $P\left(f_{c}\right)$ is a Cantor set, there is a path $\sigma$, connecting $z_{0}$ to $i_{0}$, which intersects $P\left(f_{c}\right)$ only at $i_{0}$. We can lift the path $\sigma$ to $\mathcal{N}_{c}$ to a path $\hat{\sigma}$ from a point in the fiber of $z_{0}$ to $\hat{i}$. By construction, the path $\hat{\sigma}$ intersects $\mathcal{I}_{c}$ at $\hat{i}$, hence the restriction of $\hat{\sigma}$ to $\mathcal{R}_{c}$ is a path escaping to infinity. Let $\Phi(\hat{i})=[\hat{\sigma}]$, where $[\hat{\sigma}]$ is the end represented by $\hat{\sigma}$. To check that $\Phi$ is well-defined, let $\hat{\sigma}$ and $\hat{\sigma}^{\prime}$ be two paths in $\mathcal{N}_{c}$ intersecting the irregular set only at the end point $\hat{i}$. These paths do not need to start at the same point or belong to the same leaf. Let $L$ be the leaf containing $\sigma([0,1))$ in $\mathcal{R}_{c}$. Since every leaf is dense in $\mathcal{R}_{c}$ and is simply connected, we can construct a family of paths $\hat{\sigma}_{n}$ in $L$ ending at $\hat{i}$ and such that $\hat{\sigma}_{n} \rightarrow \hat{\sigma}^{\prime}$ pointwise. Let $K$ be any compact set in $\mathcal{R}_{c}$, and $U$ be a connected component of $\mathcal{R}_{c} \backslash K$ which eventually contains $\hat{\sigma}^{\prime}$. Since $U$ is open, there is an $N$ such that $\hat{\sigma}_{N}$ also eventually belongs to $U$. But $\hat{\sigma}$ and $\hat{\sigma}_{N}$ belong to the same pathconnected component (same leaf), thus $\hat{\sigma}$ must also be eventually contained in $U$.

To see that $\Phi$ is injective, let $\hat{i}$ and $\hat{i}^{\prime}$ be two different irregular points. By Lemma 2, the projection $\pi$ is a homeomorphism between the set of irregular points and $P\left(f_{c}\right)$. Thus we have $\pi(\hat{i}) \neq \pi\left(\hat{i}^{\prime}\right)$. Hence, if $\sigma$ and $\sigma^{\prime}$ are two paths escaping to $\hat{i}$ and $\hat{i}^{\prime}$ respectively, then $\sigma$ and $\sigma^{\prime}$ eventually belong to different components of some level of renormalization. 
Finally, let us prove that $\Phi$ is surjective. Let $e$ be an end of the regular part $\mathcal{R}_{c}$, and consider a path $\hat{\sigma}$ escaping to $e$. Let $V_{0}=\overline{U_{c}(r)}$ for some $r$, where $U_{c}(r)$ is as in Section 1. For each level $n$ of renormalization, let $Z_{n}$ be the union of disjoint open neighborhoods of the little Julia sets of level $n$. If these Julia sets touch, we can shrink the domains a little to make them disjoint as in the proof of Theorem 4. Then $C_{n}=V_{0} \backslash Z_{n}$ is a compact set in $\mathbb{C} \backslash P\left(f_{c}\right)$. Thus $\pi^{-1}\left(C_{n}\right)$ is compact in $\mathcal{R}_{c}$, and by definition the path $\hat{\sigma}$ must eventually escape $\pi^{-1}\left(C_{n}\right)$. It follows that the projection $\pi(\hat{\sigma})$ eventually belongs either to a neighborhood of infinity, and then $\hat{\sigma}$ escapes to $\hat{\infty}$, or to a component of $Z_{n}$, say $\mathcal{V}_{n}$. Since the components of $Z_{n}$ are disjoint, it is clear that $\mathcal{V}_{n+1}$ is contained in $\mathcal{V}_{n}$. By a priori bounds, the domains $\left\{\mathcal{V}_{n}\right\}$ shrink to a point $i_{0}$ in $P\left(f_{c}\right)$. This process can be repeated for every coordinate of $\hat{\sigma}$ to yield a sequence of points $\left\{i_{n}\right\}$ in $P\left(f_{c}\right)$ which are the coordinates of a point $\hat{i}$ in $\widehat{P\left(f_{c}\right)}$. By Lemma $2, \hat{i}$ is irregular. Now, local neighborhoods of irregular points in $\mathcal{N}_{c}$ intersect complements of compact sets in $\mathcal{R}_{c}$. So one can check that $\Phi$ induces the desired homeomorphism.

In the remaining part of the paper, $h$ will denote a homeomorphism of the regular parts of two infinitely renormalizable quadratic polynomials, $f_{c}$ and $f_{c^{\prime}}$, with a priori bounds.

COROllary 16. The map $h$ admits an extension to a homeomorphism $\tilde{h}: \mathcal{N}_{c} \rightarrow \mathcal{N}_{c^{\prime}}$ of the natural extensions. Moreover, $\tilde{h}(\hat{\infty})=\hat{\infty}$.

Proof. By Proposition 15 the map $h$ extends to the natural extensions sending irregular points to irregular points, and by Lemma 2 the point $\hat{\infty}$ is the only isolated irregular point, hence $h(\hat{\infty})=\hat{\infty}$.

4.3. Topology of periodic leaves. Since leaves are path-connected components of $\mathcal{R}_{c}$, given a leaf $L \subset \mathcal{R}_{c}$ we can consider how many accesses to $\hat{\infty}$ the leaf has, that is, the number of path components of $L \backslash K$ that are connected to $\hat{\infty}$ in $\mathcal{N}_{c}$, for a suitable large compact set $K \subset \mathcal{R}_{c}$. Note that a leaf has access to a point in $P\left(f_{c}\right)$ if and only if it intersects infinitely many levels in the tree structure of $\mathcal{R}_{c}$. However, this is not the case for repelling leaves:

Lemma 17. Let $L$ be a repelling leaf. Then there is a level $n$ such that $L \subset \mathcal{Q}_{n}$. In this case, $L$ has access only to $\hat{\infty}$.

Proof. Let $\hat{p}$ be the periodic point in $L$ and let $p=\pi(\hat{p})$. Since $f_{c}$ is infinitely renormalizable, $p$ is repelling, and therefore it must belong to the Julia set $J\left(f_{c}\right)$. Moreover, the inverse of the classical Königs linearization coordinate around $p$ provides a global uniformization coordinate for $L$. From this uniformization it follows that a point $\hat{z}$ in $\mathcal{R}_{c}$ belongs to $L$ only if the coordinates of $\hat{z}$ accumulate on the cycle of $p$. 
Since the postcritical set is the intersection of the renormalization domains, we can find a level $n+1$ of the renormalization such that the orbit, of renormalization domains of level $n+1$, is outside a neighborhood of the cycle of $p$. By this choice, no point in $L$ can intersect the level $n+1$ of the tree structure of $\mathcal{R}_{c}$. The statement of the lemma now follows.

Let $f_{s}$ be a superattracting map; then every leaf $L$ invariant under some iterate of $\hat{f}_{s}$ must contain a repelling periodic point and hence $L$ is repelling. In this case, there are no critical points in the Julia set $J\left(f_{s}\right)$ so the fiber $\pi^{-1}\left(J\left(f_{s}\right)\right)$ is compact (see [16, Lemma 8.4]). For a periodic point $p$ in $J\left(f_{s}\right)$, let $\hat{p}$ be an invariant lift of $p$ in $\mathcal{R}_{s}$. From [3], we have the following:

Proposition 18. Let $f_{s}$ be a superattracting polynomial, and let $L$ be a repelling leaf in $\mathcal{R}_{s}$. Then the number of accesses of $L$ to $\hat{\infty}$ is equal to the number of external rays landing at $p$. Moreover, if $L$ is a leaf which has at least three accesses to ends, then $L$ must be repelling.

Let us remark that Proposition 15 also holds when $f_{s}$ is superattracting, however, repelling leaves may have access to other irregular points. Nevertheless, if some repelling leaf $L$ has at least three accesses to $\hat{\infty}$ then by Proposition 18, the corresponding periodic point $p$ has at least three external rays landing at $p$. If $f_{c}$ is non-hyperbolic, then there exists a periodic point $p$ with at least three rays landing at $p$ if and only if $\operatorname{Im}(c) \neq 0$.

Let us now go back to the case where $f_{c}$ is infinitely renormalizable with a priori bounds:

Lemma 19. Let $f_{c}$ be infinitely renormalizable with a priori bounds. Let $L \subset \mathcal{R}_{c}$ be a leaf that has access to $\hat{\infty}$ but to no other irregular point, and the number of accesses to infinity is at least three. Then $L$ must be a repelling leaf.

Proof. Since the only access to infinity of $L$ is $\hat{\infty}$, there is a level $n$ such that $L \subset \mathcal{Q}_{n}$. Recall, as in the discussion prior to Proposition 6, that the dynamics of $\hat{f}_{c}$ on $\mathcal{Q}_{n}$ determines a superattracting polynomial $f_{\sigma_{n}}$. By Proposition 6, $\mathcal{Q}_{n}$ is homeomorphic to $\mathcal{Q}_{\sigma_{n}}$. Let $L^{\prime}$ be the leaf in $\mathcal{Q}_{\sigma_{n}}$ corresponding to $L$. By Proposition 18, the leaf $L^{\prime}$ is repelling in $\mathcal{Q}_{\sigma_{n}}$ under dynamics of $\hat{f}_{\sigma_{n}}$ and has at least three accesses to $\hat{\infty}$. Let $\hat{p}^{\prime}$ be the periodic point in $L^{\prime}$ under $\hat{f}_{\sigma_{n}}$. Then $p^{\prime}:=\pi\left(\hat{p}^{\prime}\right)$ is a periodic point in $J\left(f_{\sigma_{n}}\right)$ such that there are at least three rays landing at $p^{\prime}$ by the construction of the homeomorphism between $\mathcal{Q}_{n}$ and $\mathcal{Q}_{\sigma_{n}}$. Now, there exists a periodic point $p$ in $J\left(f_{c}\right)$ whose ray portrait is the same as that of $p^{\prime}$. In particular, $p$ has at least three rays landing. Let $Z_{n}$ be as in the proof of Proposition 15. Then $p \in \mathbb{C} \backslash Z_{n}$ and $L$ contains a periodic lift of $p$. Thus $L$ is repelling. 
Now we are ready to prove Theorem 7:

Proof of the Main Theorem. By Corollary 16, the map $h$ extends to a homeomorphism of natural extensions $\tilde{h}$, with $h(\hat{\infty})=\hat{\infty}$. Since $\operatorname{Im}(c) \neq 0$, there exists a repelling leaf $L$ in $\mathcal{N}_{c}$ such that $L$ has at least three accesses to $\hat{\infty}$. This is a topological property, so $h(L)$ is also a leaf with at least three accesses to $\hat{\infty}$. By Lemma 19, $h(L)$ is also repelling and moreover $\operatorname{Im}\left(c^{\prime}\right) \neq 0$. In this way, $\tilde{h}$ sends a repelling leaf into a repelling leaf. By an isotopy argument similar to the one used in the proof of Lemma 14, we can see that this implies that $h$ sends repelling leaves into repelling leaves. Hence, $\tilde{h}$ satisfies the conditions of Theorem 9 , which implies that $f_{c}$ and $f_{c^{\prime}}$ belong to the same combinatorial class.

Acknowledgments. We would like to thank for the hospitality and support of the Fields Institute and IMPAN (Warsaw) where this work was carried out. We would also want to thank Misha Lyubich for useful conversations. The first author wants to thank IMATE (Cuernavaca) for the hospitality and support. The second author appreciates the research grants by JSPS Grant-in-Aid for Young Scientists, the Circle for the Promotion of Science and Engineering, and Inamori Foundation.

\section{References}

[1] H. Bruin and D. Schleicher, Symbolic dynamics of quadratic polynomials, MittagLeffler Preprint Ser. 2002.

[2] C. Cabrera, Towards classification of laminations associated to quadratic polynomials, Ph.D. thesis, Stony Brook Univ., 2005, http://www.math.sunysb.edu/cgibin/thesis.pl?thesis05-3.

[3] -, On the classification of laminations associated to quadratic polynomials, J. Geom. Anal. 18 (2008), 29-67.

[4] A. Douady et J. H. Hubbard, Étude dynamique des polynômes complexes I, II, Publ. Math. Orsay 84-2, 85-4, Dép. Math. Univ. Paris-Sud, 1984, 1985.

[5] J. H. Hubbard and R. W. Oberste-Vorth, Hénon mappings in the complex domain. II. Projective and inductive limits of polynomials, in: Real and Complex Dynamical Systems (Hillerød, 1993), NATO Adv. Sci. Inst. Ser. C Math. Phys. Sci. 464, Kluwer, Dordrecht, 1995, 89-132.

[6] J. Kahn, A priori bounds for some infinitely renormalizable quadratics. I. Bounded primitive combinatorics, arXiv:math.DS/0609045, 2006.

[7] J. Kahn and M. Lyubich, A priori bounds for some infinitely renormalizable quadratics. II. Decorations, Ann. Sci. École Norm. Sup. (4) 41 (2008), 57-84.

[8] V. Kaimanovich and M. Lyubich, Conformal and harmonic measures on laminations associated with rational maps, Mem. Amer. Math. Soc. 173 (2005), no. 820.

[9] T. Kawahira, On the regular leaf space of the cauliflower, Kodai Math. J. 26 (2003), 167-178.

[10] -, Tessellation and Lyubich-Minsky laminations associated with quadratic maps II: Topological structures of 3-laminations, Conform. Geom. Dynam. 13 (2009), 6-75. 
[11] J. Kiwi, Rational laminations of complex polynomials, in: Laminations and Foliations in Dynamics, Geometry and Topology (Stony Brook, NY, 1998), Contemp. Math. 269, Amer. Math. Soc., Providence, RI, 2001, 111-154.

[12] J. Kwapisz, Homotopy and dynamics for homeomorphisms of solenoids and Knaster continua, Fund. Math. 168 (2001), 251-278.

[13] M. Lyubich, Dynamics of the rational transforms: the topological picture, Uspekhi Mat. Nauk 41 (1986), no. 4, 35-95 (in Russian); English transl.: Russian Math. Surveys 41 (1986), 43-117.

[14] -, Combinatorics, geometry and attractors of quasi-quadratic maps, Ann. of Math. (2) 140 (1994), 347-404.

[15] -, Dynamics of quadratic polynomials. I, II, Acta Math. 178 (1997), 185-247, $247-297$.

[16] M. Lyubich and Y. Minsky, Laminations in holomorphic dynamics, J. Differential Geom. 47 (1997), 17-94.

[17] C. McMullen, Complex Dynamics and Renormalization, Ann. of Math. Stud. 135, Princeton Univ. Press, Princeton, NJ, 1994.

[18] J. Milnor, Local connectivity of Julia sets: expository lectures, in: The Mandelbrot Set, Theme and Variations, London Math. Soc. Lecture Note Ser. 274, Cambridge Univ. Press, Cambridge, 2000, 67-116.

[19] - Periodic orbits, external rays and the Mandelbrot set: an expository account, Astérisque 261 (2000), 277-333.

[20] D. Sullivan, Bounds, quadratic differentials, and renormalization conjectures, Amer. Math. Soc. Centennial Publications, Vol. II (Providence, RI, 1988), Amer. Math. Soc. Providence, RI, 1992, 417-466.

Carlos Cabrera

Instituto de Matemáticas

Unidad Cuernavaca. UNAM

Av. Universidad s/n, Col. Lomas de Chamilpa

C. P. 62210, Cuernavaca, Morelos, Mexico

E-mail: carlos@matcuer.unam.mx
Tomoki Kawahira Graduate School of Mathematics

Nagoya University

Nagoya 464-8602, Japan

E-mail: kawahira@math.nagoya-u.ac.jp

Received 14 July 2008;

in revised form 27 March 2009 